\title{
Provision of chlamydia testing in a nationwide service offering termination of pregnancy: with data capture to monitor prevalence of infection
}

\author{
H Mallinson, J Hopwood, S Skidmore, K Fenton, C Phillips, I Jones
}

Sex Transm Infect 2002;78:416-421

See end of article for authors' affiliations

\section{Correspondence to} Dr Harry Mallinson, PHLS Liverpool, University Hospital at Aintree, Lower Lane, Liverpool L9 7AL, UK;

hmallinson@nw.phls.nhs.uk

Accepted for publication 18 July 2002

\begin{abstract}
Objectives: To establish a methodology by which all women attending for termination of pregnancy (TOP) at British Pregnancy Advisory Service (BPAS) branches may be approached to participate in Chlamydia trachomatis screening. To examine the feasibility of monitoring $C$ trachomatis prevalence and the impact of charging for screening on the uptake rate in this population.

Methods: Patients attending for TOP at participating BPAS branches were offered a test for chlamydia infection and asked to complete a questionnaire. Urine samples from participants were tested using a nucleic acid amplification test (NAAT).

Results: 1001 women provided a urine sample, a 77\% response rate among those participating in the study. Factors significantly associated with taking up chlamydia screening included symptoms, previous TOP, parity, and no previous chlamydial test. Overall prevalence of genital chlamydial infection was $7.5 \%$, with highest age specific prevalences occurring among attendees aged 20-24 years (11.5\%) and under 20 years $(10.8 \%)$. In univariate analysis, chlamydia positivity was significantly associated with respondent age and previous diagnosis with chlamydia. Only 35\% of women who had the screening test would have done so had they been asked to pay the $£ 20$ clinical, administrative, and laboratory costs of the examination.

Conclusions: We have demonstrated the feasibility of routine chlamydia screening and the potential for prospective prevalence monitoring across the nationwide BPAS service. In most cases the chlamydia result was available within the clinical contact period for the TOP. Charging patients directly for the test could reduce uptake of chlamydia screening to levels unsatisfactory for both the public health and prevalence monitoring purposes.
\end{abstract} ally transmitted infection in the United Kingdom. ${ }^{1}$ Increasing incidence, prevalence, and associated adverse outcomes including pelvic inflammatory disease, infertility, and ectopic pregnancy have resulted in its being designated a major public health concern. It has been recommended that women seeking TOP should be tested for chlamydia because they have high prevalence ${ }^{2}$ and because termination procedures may increase the risk of ascending upper genital tract infection. ${ }^{3}$

Studies in the United States have detected decreases in chlamydia prevalence among TOP clinic attendees following the introduction of population based screening programmes. There is evidence from Norway that the high disease prevalence and the stable nature of the study population (TOP clinic attendees) can provide a good approach to monitor trend. ${ }^{4}$ Monitoring chlamydia in a nationwide termination service could provide useful sentinel data about changes in the prevalence of the infection in the community as national control programmes for chlamydia are introduced and evaluated. Also, since approximately $20 \%$ of TOP patients return for subsequent terminations, information on incidence and reinfection in this population would become available.

The British Pregnancy Advisory Service (BPAS) is a nationwide charitable sector termination service with common standards, protocols, and data collection throughout its 40 branches (sites for assessment for termination of pregnancy) and eight clinics (where treatment is carried out). Clients (about 50000 per annum) may be self funded (40\%) or seen under one of a variety of NHS contractual agreements (60\%). Some of the authors ( JH, HM, IJ) have previously published an evaluation of chlamydia screening feasibility and test methods at BPAS sites. ${ }^{5}$ The aim of the current project was to implement a chlamydia screening strategy within BPAS branches and clinics in order to determine the prevalence of $C$ trachomatis infection in this patient population using routinely gathered data. Such baseline prevalence information is essential for informing the planning and prioritisation of chlamydia intervention programmes being considered by the Department of Health.

All women attending BPAS for a TOP are currently given prophylactic antibiotic treatment to reduce risk of ascending reproductive tract infection. Thus, addition of screening for chlamydia in these venues is a public health measure designed to facilitate detection of asymptomatic chlamydial infection among patients and, indirectly, their partners. Consequently it is important to consider how testing in this setting should be funded and whether it is ethical or acceptable to ask clients for direct payment. This paper examines the prevalence of chlamydia among TOP clinic attenders, the factors associated with chlamydia positivity within this group, and the potential influence of charging for screening tests on their uptake.

\section{METHODS}

Setting

Participating BPAS branches included Birmingham Central, Cannock, Coventry, Telford (Midlands) and Chester, Liverpool, Preston, Manchester, and Sheffield (north of England). These locations are representative in size and staffing of those covered by BPAS nationally.

Patient recruitment and specimen collection

Detailed procedures for introducing the study and inviting patients to participate were designed. Patients (private and 
NHS) who attended for assessment for TOP were offered a test for chlamydial infection. There was no restriction on age and, as it was considered unethical to randomise patients into payment and non-payment groups, all patients were offered the screening test free of charge. The study rationale and procedures were explained to all patients. Verbal informed consent was obtained from all those who agreed to participate. Patients accepting the test were asked to provide a "first catch" urine (FCU) specimen collected after at least 1 hour of urine retention. The sample was collected in a plastic "one ounce" bottle. Patients, whether accepting the test or not, were asked to complete (alone or assisted by a healthcare worker) a questionnaire incorporated into the laboratory request form. This obtained demographic, clinical, and behavioural information and included a means, agreeable to the patient, of communicating the test result. Where a patient declined a urine test, the reason (outright refusal or a previous test) was recorded if given. A question asked respondents whether they would still have had the test if it cost $£ 20$ (representing laboratory charges and BPAS administration and patient management costs).

\section{Specimen transport and testing}

Urine samples were stored at $4-8^{\circ} \mathrm{C}$ before transportation by local courier to the nearest PHLS laboratory then, if necessary, forwarded overnight by PHLS transport at ambient temperature to the testing site (Liverpool PHL or Telford PHL). On receipt all samples were tested using a nucleic acid amplification test-the ligase chain reaction (LCR) system (Abbott Laboratories) which was used according to the manufacturers instructions. To avoid any delay in transmission of results these were sent, on the day generated, to a secure fax machine at the relevant BPAS clinic - that is, the location at which the TOP would be performed. To maintain confidentiality, only the patient's BPAS number, date of birth, and operation date were used as identifiers. Full printed reports followed by post.

\section{Management of chlamydia positive women}

All women with a chlamydia positive LCR result were managed in accordance with the study protocol which included treatment with a single $1 \mathrm{~g}$ dose of azithromycin. Positive results were generally given at the clinic, on the day of the TOP, by a clinic healthcare worker who also gave general information on chlamydia. All chlamydia positive patients were referred, with a letter, to their local genitourinary medicine (GUM) clinic for further management and partner notification.

The information pack provided for the patient included further information about chlamydia, two timetables of opening hours-one of which was for the partner, and introduction slips for the local GUM clinic. In addition, BPAS staff completed a checklist for each patient to ensure that the following were addressed: antibiotics given or referral for treatment, information given about chlamydia either verbal or written, importance of partner(s) being treated, discussion of no sex until partners are cured, referral letter to GUM department given.

\section{Data collection, transfer, and analysis}

A specially designed laboratory request form/questionnaire was used which was similar to that developed by some of us $(\mathrm{JH}, \mathrm{HM})$ for use in the NHS Chlamydia Pilot Screening Programme. ${ }^{6}$ The form consisted of a main portion (collecting core demographic and relevant clinical data) with a detachable key to response boxes for more detailed behavioural, service utilisation, and risk factor information. It was anticipated that all patient based data (clinical, demographic, and behavioural) required for the project would be obtained on the laboratory request form and that the detachable key would obscure sensitive information in transit. Data entry was carried out by appropriate staff at the testing laboratories. Data were initially stored in secure (password protected) laboratory database systems (TELEPATH )

Analysis was performed using STATA version 6.0. Overall prevalence of $C$ trachomatis infection (main outcome measure) was estimated from women testing LCR positive for infection as a proportion of all women providing a FCU sample. Age specific prevalence for $C$ trachomatis was calculated. Testing site, demographic, and behavioural variations in chlamydia prevalence were also explored. The $\chi^{2}$ test or Fisher's exact test was used to explore associations between factors and whether a sample was provided and its result. Significant associations found in the single variable analysis with respect to the prevalence of $C$ trachomatis infection were explored in a multivariable logistic regression model to determine the factors significantly and independently associated with being diagnosed with $C$ trachomatis infection.

\section{Ethics}

Approval was obtained from the ethics committees of both BPAS and PHLS.

\section{RESULTS}

A total of 1293 women participated in the study and completed the questionnaire. Of these, 292 declined testing ( 84 because of a previous test) and 1001 (77\%) provided a urine sample. Comparing women who accepted or declined the urine test, there were no significant differences with respect to age, ethnicity, or recorded partner change in the past year (see table 1). However, significant differences were found in their chlamydia testing and diagnosis history, symptomatology, and reproductive history. Women who accepted the urine test were less likely to have been previously tested for chlamydia $(7.7 \%$ v $28.1 \%$. $\mathrm{p}<0.001)$ and less likely to have had a previously positive chlamydia test $(1.8 \% \vee 6.6 \%, \mathrm{p}<0.001)$. They were more likely to be symptomatic $(39.6 \% \vee 7.8 \%$ reporting at least one symptom, $<<0.001$ ) and were also likely to have had more children, miscarriages, and previous TOP.

\section{Prevalence of $\mathbf{C}$ trachomatis}

Three patients provided insufficient urine for LCR testing. Of the remaining 998, 75 (7.5\%) were found positive for $C$ trachomatis infection. At the four Midlands branches a total of 505 urines were tested and at the six north of England branches 493 urines were tested with positive rates of $7.5 \%$ overall in both regions. At the five branches which collected over 100 samples each, the positivity ranged from $5.8 \%$ to $8.2 \%$. Chlamydia positivity was significantly associated with younger age $(p<0.001)$, having previously tested positive $(p=0.02)$, and having been previously treated $(p=0.01)$ for genital chlamydia. Age specific prevalence ranged from 1.8\% among those over 35 years to $11.5 \%$ among those aged between 20-24 years (table 2). Relatively high disease prevalence $(10.8 \%)$ was also observed among those aged under 20 years. Seventy seven of the women tested reported a previous chlamydia test. Those who had a previous chlamydia test were equally likely to have chlamydia diagnosed compared with those who had never been tested previously $(7.8 \% v 7.5 \%, p=0.9)$. However, women who had previously been diagnosed with chlamydia were significantly more likely to be chlamydia positive than those who had tested negative previously, as well as those who had never been previously tested $(23.5 \% v 0 \%$ and $7.5 \%$ respectively $\mathrm{p}=0.02)$. A chlamydia prevalence of $20 \%(3 / 15)$ among women who had been previously treated for chlamydia raises questions about adequacy of treatment or reinfection.

We found no association between chlamydia positivity and ethnicity, nor with women's willingness to pay for chlamydia testing. Neither was there any association between positivity and the presence of any reported clinical symptom(s), nor 
Table 1 Characteristics among women accepting or declining a chlamydia test

\begin{tabular}{|c|c|c|c|}
\hline & $\begin{array}{l}\text { Women accepting } \\
\text { urine test (n) }\end{array}$ & $\begin{array}{l}\text { Women declining } \\
\text { urine test (n) }\end{array}$ & Significance test \\
\hline Base & 1001 & 292 & \\
\hline Age group & $(n=1001)$ & $(n=290)$ & \\
\hline$<19$ & $24.1 \%(241)$ & $20.7 \%(60)$ & $p=0.15$ \\
\hline $20-24$ & $28.8 \%(288)$ & $24.8 \%(72)$ & \\
\hline $25-34$ & $36.1 \%(361)$ & $40.3 \%(117)$ & \\
\hline$>35$ & $11.1 \%(111)$ & $14.1 \%(41)$ & \\
\hline Ethnicity & $(n=897)$ & $(n=221)$ & \\
\hline White & $91.2 \%(818)$ & $92.3 \%(204)$ & $p=0.78$ \\
\hline Black & $3.8 \%(34)$ & $4.1 \%(9)$ & \\
\hline Asian & $3.9 \%(35)$ & $3.2 \%(7)$ & \\
\hline Other & $1.1 \%(10)$ & $0.5 \%(1)$ & \\
\hline Ever had previous chlamydia test & $(n=1001)$ & $(n=292)$ & \\
\hline Yes & $7.7 \%(77)$ & $28.1 \%(82)$ & $p<0.001$ \\
\hline No & $92.3 \%(924)$ & $71.9 \%(210)$ & \\
\hline Ever had previous positive chlamydia test & $(n=964)$ & $(n=229)$ & \\
\hline Yes & $1.8 \%(17)$ & $6.6 \%(15)$ & $p<0.001$ \\
\hline No & $2.4 \%(23)$ & $1.8 \%(4)$ & \\
\hline Never tested & $95.9 \%(924)$ & $91.7 \%(210)$ & \\
\hline Number of symptoms & $(n=1001)$ & $(n=292)$ & \\
\hline 0 & $60.7 \%(608)$ & $92.1 \%(269)$ & $p<0.001$ \\
\hline 1 & $25.7 \%(257)$ & $6.9 \%(20)$ & \\
\hline 2 & $10.0 \%(100)$ & $0.3 \%(1)$ & \\
\hline 3 & $3.0 \%(30)$ & $0.3 \%(1)$ & \\
\hline 4 & $0.9 \%(6)$ & $0.3 \%(1)$ & \\
\hline Partner change in the last year & $(n=654)$ & $(n=67)$ & \\
\hline Yes & $17.3 \%(113)$ & $9.0 \%(6)$ & $p=0.08$ \\
\hline No & $82.7 \%(541)$ & $91.0 \%(61)$ & \\
\hline Number of children & $(n=1001)$ & $(n=292)$ & \\
\hline 0 & $58.3 \%(584)$ & $81.5 \%(238)$ & $p<0.001$ \\
\hline 1 & $17.3 \%(173)$ & $7.5 \%(22)$ & \\
\hline 2 & $15.9 \%(159)$ & $8.2 \%(24)$ & \\
\hline 3 or more & $8.5 \%(85)$ & $2.7 \%(8)$ & \\
\hline Number of miscarriages & $(n=1001)$ & $(n=292)$ & \\
\hline 0 & $88.0 \%(881)$ & $95.6 \%(279)$ & $p=0.001$ \\
\hline 1 & $9.7 \%(97)$ & $4.1 \%(12)$ & \\
\hline 2 or more & $2.3 \%(23)$ & $0.3 \%(1)$ & \\
\hline Number of previous TOP & $(n=1001)$ & $(n=292)$ & \\
\hline 0 & $75.2 \%(753)$ & $92.5 \%(270)$ & $p<0.001$ \\
\hline 1 & $20.7 \%(207)$ & $5.8 \%(17)$ & \\
\hline 2 or more & $4.1 \%(41)$ & $1.7 \%(5)$ & \\
\hline
\end{tabular}

with the number of symptoms reported by the patient. A single question exploring sexual partner change and chlamydia positivity was included in the questionnaire; however, its completion rate was poor (65\% provided a response). Chlamydia prevalence among those who reported having changed a partner in the previous year was $6.2 \%$ (7/113) compared with $7.6 \%$ (41/538) among women who had not done so. There were no significant associations between chlamydia positivity and numbers of children $(p=0.8)$, number of previous miscarriages $(p=0.6)$, and previous termination of pregnancy $(\mathrm{p}=0.2)$.

\section{Factors associated with $\mathrm{C}$ trachomatis positivity}

Those factors exhibiting evidence of an association $(p \leqslant 0.2)$ with chlamydia positivity-age, ethnicity, previous positive test, previous treated infection, irregular bleeding, and the number of previous TOP-were included in a multivariable logistic regression analysis to find those factors independently associated with chlamydia positivity using a backward selection approach to obtain a final model. Four factors-age $(p=0.002)$, ethnicity $(p=0.01)$, previous positive test $(\mathrm{p}=0.06)$, and irregular bleeding $(\mathrm{p}=0.05)$-remained in the model. All possible two way interactions between these factors were tested but none was significant.

The impact of charging for chlamydia screening

Women were asked whether they would have consented to a chlamydia test if they were to be charged $£ 20$ (table 3). Overall, 35\% (range $8-51 \%$ across branches) of women who provided a urine sample indicated that they would still have requested the test.

\section{The feasibility of $\mathrm{C}$ trachomatis testing in BPAS sites}

A number of process measures were included to explore the efficiency and effectiveness of specimen transfer, testing, and reporting between BPAS sites and the public health laboratories. Measured indicators (table 3 ) included the proportion of test results reaching the clinic by the operation date (reporting delay) and the proportion of samples reaching the laboratory after the operation date (specimen transfer delay). Only $4.6 \%$ (range $0-70 \%$ ) of samples arrived at the laboratory after the operation date and $86.2 \%$ (range $30-95.4 \%$ ) of results arrived at clinics by the operation date. 
Table 2 Chlamydia prevalence by key demographic and clinical variables

\begin{tabular}{|c|c|c|c|}
\hline & $\begin{array}{l}\text { Chlamydia prevalence } \\
(\mathrm{N} / \mathrm{N})\end{array}$ & $\begin{array}{l}\text { Relative risk ( } 95 \% \\
\text { confidence interval) }\end{array}$ & $\mathrm{p}$ Value \\
\hline Base & 998 & & \\
\hline Age group & $(n=998)$ & & \\
\hline$<20$ & $10.8 \%(26 / 240)$ & 1 & $<0.001 *$ \\
\hline $20-24$ & $11.5 \%(33 / 288)$ & $1.06(0.65$ to 1.72$)$ & \\
\hline $25-34$ & $3.9 \%(14 / 360)$ & $0.36(0.19$ to 0.67$)$ & \\
\hline$>34$ years & $1.8 \%(2 / 110)$ & $0.17(0.04$ to 0.69$)$ & \\
\hline Race/ethnicity & $(n=896)$ & & \\
\hline White & $8.1 \%(66 / 817)$ & 1 & 0.1 \\
\hline Black & $0 \%(0 / 34)$ & $0(-)$ & \\
\hline Asian & $0 \%(0 / 35)$ & $0 \mathrm{H}-1$ & \\
\hline Other & $10.0 \%(1 / 10)$ & $1.24(0.19$ to 8.06$)$ & \\
\hline Willing to pay $£ 20$ for test & $(n=998)$ & & \\
\hline No & $8.1 \%(53 / 653)$ & 1 & 0.3 \\
\hline Yes & $6.4 \%(22 / 345)$ & 0.79 (0.49 to 1.27$)$ & \\
\hline Previous chlamydia test & $(n=998)$ & & \\
\hline No & $7.5 \%(69 / 921)$ & 1 & 0.9 \\
\hline Yes & $7.8 \%(6 / 77)$ & $1.04(0.47$ to 2.31$)$ & \\
\hline Previous positive chlamydia test & $(n=961)$ & & \\
\hline Yes & $23.5 \%(4 / 17)$ & 1 & 0.02 \\
\hline No & $0 \%(0 / 23)$ & $0.00(-)$ & \\
\hline Never tested & $7.5 \%(69 / 921)$ & 0.32 (0.13 to 0.77$)$ & \\
\hline Previous treated infection & $(n=998)$ & & \\
\hline Yes & $20.0 \%(3 / 15)$ & 1 & 0.01 \\
\hline Unknown & $50.0 \%(1 / 2)$ & $2.50(0.45$ to 13.91$)$ & \\
\hline Not applicable & $7.2 \%(71 / 981)$ & $0.36(0.12$ to 1.02$)$ & \\
\hline Partner change in last year & $(n=651)$ & & \\
\hline No & $7.6 \%(41 / 538)$ & 1 & 0.6 \\
\hline Yes & $6.2 \%(7 / 113)$ & 1.23 (0.57 to 2.67$)$ & \\
\hline Vaginal discharge & $(n=760)$ & & \\
\hline No & $7.8 \%(41 / 525)$ & 1 & 0.5 \\
\hline Yes & $9.4 \%(22 / 235)$ & 1.20 (0.73 to 1.97$)$ & \\
\hline Pelvic pain & $(n=712)$ & & \\
\hline No & $8.3 \%(48 / 581)$ & 1 & 0.6 \\
\hline Yes & $6.9 \%(9 / 131)$ & $0.83(0.42$ to 1.65$)$ & \\
\hline Cystitis & $(n=692)$ & & \\
\hline No & $8.8 \%(51 / 582)$ & 1 & 0.4 \\
\hline Yes & $6.4 \%(7 / 110)$ & $0.74(0.35$ to 1.60$)$ & \\
\hline Irregular bleeding & $(n=675)$ & & \\
\hline No & $8.76(51 / 582)$ & 1 & 0.1 \\
\hline Yes & $4.3 \%(4 / 93)$ & $0.49(0.18$ to 1.32$)$ & \\
\hline Number of symptoms*** & $(n=998)$ & & \\
\hline 0 & $6.4 \%(45 / 606)$ & 1 & $0.96 * *$ \\
\hline 1 & $7.8 \%(20 / 257)$ & $1.05(0.63$ to 1.74$)$ & \\
\hline 2 & $8.1 \%(8 / 99)$ & $1.09(0.53$ to 2.24$)$ & \\
\hline 3 & $6.7 \%(2 / 30)$ & $0.90(0.23$ to 3.53$)$ & \\
\hline 4 & $0 \%(0 / 6)$ & $0.00(-)$ & \\
\hline Number of children & $(n=998)$ & & \\
\hline 0 & $7.7 \%(45 / 584)$ & 1 & 0.8 \\
\hline 1 & $8.7 \%(15 / 172)$ & $1.13(0.65$ to 1.98$)$ & \\
\hline 2 & $6.4 \%(10 / 157)$ & $0.93(0.43$ to 1.60$)$ & \\
\hline 3 or more & $5.9 \%(5 / 85)$ & $0.76(0.31$ to 1.87$)$ & \\
\hline Number of miscarriages & $(n=998)$ & & \\
\hline 0 & $7.4 \%(65 / 880)$ & 1 & 0.6 \\
\hline 1 & $9.5 \%(9 / 95)$ & $1.28(0.66$ to 2.49$)$ & \\
\hline 2 or more & $4.4 \%(1 / 23)$ & 0.59 (0.09 to 4.06$)$ & \\
\hline Number of previous TOP & $(n=998)$ & & \\
\hline 0 & $8.4 \%(63 / 752)$ & 1 & 0.2 \\
\hline 1 & $4.4 \%(9 / 206)$ & $0.52(0.26$ to 1.03$)$ & \\
\hline 2 or more & $7.5 \%(3 / 40)$ & $0.90(0.29$ to 2.73$)$ & \\
\hline
\end{tabular}

${ }^{*} \chi^{2}$ test of linear trend $p<0.001$, departure from linear trend $p=0.1$

$* \star \chi^{2}$ test of linear trend $p=0.9$, departure from linear trend $p=0.9$

$* * \star$ Number of symptoms reported, ie if entry was missing or unknown then no symptom assumed. 
Table 3 Process indicators related to the receipt, reporting and the acceptability of charging for a screening test for $C$ trachomatis infection among TOP clinic attenders

\begin{tabular}{llll}
\hline BPAS branch & $\begin{array}{l}\text { Percentage of samples } \\
\text { reaching the laboratory } \\
\text { after the day of TOP }\end{array}$ & $\begin{array}{l}\text { Percentage of results } \\
\text { reaching clinic by the } \\
\text { day of TOP procedure }\end{array}$ & $\begin{array}{l}\text { Percentage of women who } \\
\text { would still agree to screen if } \\
\text { charged at £20 }\end{array}$ \\
\hline A & 7.7 & 91.6 & 19 \\
B & 70.0 & 30.0 & 10 \\
C & 0.0 & 83.8 & 41 \\
D & 3.1 & 95.4 & 51 \\
E & 3.8 & 55.0 & 8 \\
F & 1.2 & 88.9 & 40 \\
G & 0.0 & 83.3 & 19 \\
H & 0.0 & 60.0 & 36 \\
J & 7.1 & 85.7 & 38 \\
Overall & 4.6 & 86.2 & 35 \\
\hline
\end{tabular}

\section{DISCUSSION}

This study examined the feasibility and outcomes of undertaking urinary screening for $C$ trachomatis infection at BPAS branches and explored the possible impact of charging for this service. The high prevalence of chlamydial infection among attendees ( $11.2 \%$ in those below 25 years of age) confirms the vulnerability of this clinic population and is consistent with findings from other developed countries. ${ }^{7-9}$ Examination of risk factors enables the identification of possible confounders of analysis for surveillance purposes (for example, age, previous diagnosis of chlamydia). Prospective monitoring of chlamydia positivity in this setting may be used to reflect the impact of chlamydia screening in primary care sites as advocated ${ }^{2}$ by the chief medical officer (CMO), or other community based sexual health interventions. In a wider context, changes in chlamydia prevalence may parallel changes in general sexual health of a population allowing feedback to health authorities/primary care groups on the effects of local implementation of sexual health strategies. The level of sampling achieved suggests that representative and consistent data can be obtained.

Screening for chlamydial infection in women seeking termination of pregnancy has been recommended ${ }^{2}$ and it will be important to develop a framework through which the prevalence of $C$ trachomatis infection among service users may be monitored. Our study highlighted the feasibility of coordinating chlamydia screening within the current TOP procedures. We are encouraged that very few samples experienced significant transport delay to the laboratory and that the majority of clinics received the results in time for the procedure. Clearly, any proposed screening programme within BPAS sites would need to be funded and one strategy would be to ask women themselves to pay for the chlamydia testing. Our study suggests that many women would be unwilling or unable to do so. Just over a third of women would be willing to have the chlamydia test if they were requested to pay $£ 20$. Although there were no recorded significant differences between those who were willing to pay and those who were not, failure to screen $65 \%$ of women would be unsatisfactory for both public health and prevalence monitoring purposes. We would strongly encourage that chlamydia testing is offered free of charge to patients utilising the services of BPAS. The public health benefits equate to those from the wider screening of the population, as recommended by the CMO, and should be funded similarly.

A key consideration for successful implementation of a TOP clinic based screening programme is its acceptability to staff. Although not formally evaluated in this study, we obtained and documented the informal feedback of clinical colleagues who were involved in the implementation and delivery of this study. BPAS staff preferred the model used-namely, urine testing at the branch with results available at the clinic, to other possible alternatives that included near patient testing at the clinic (some clinics had no on-site laboratory capability and there were time constrains in having the result available), or sending swabs to the microbiology laboratory at the time of the operation (no opportunity to discuss results and implications with the patient).

There are a few limitations to our study. The lack of association between recent sexual partner change and chlamydia positivity, in our study, in part reflects the poor completion of this item by clinic staff. This resulted from some misunderstanding and embarrassment experienced by patients and clinic staff. Secondly, although every effort was made to ensure that results were received at clinics before the TOP date, this was not possible in some cases. The poorer performance figures relating to tests undertaken at branches $\mathrm{B}$ and $\mathrm{H}$ were due to misunderstandings in transport arrangements and could easily be addressed. The low (55\%) achievement for results reaching the clinic by the day of operation for patients tested at branch $\mathrm{E}$ reflects the higher proportion of patients who can be offered an operation date closer to their consultation visit at that particular BPAS site. Finally, all patients diagnosed with chlamydia in our study were referred to a GUM clinic in case of other concurrent infections and for partner notification (a key secondary prevention activity aimed at reducing onward transmission and potential complications of undiagnosed disease) but we did not collect information on these outcomes.

This evaluation focused on the feasibility of undertaking routine chlamydia screening among BPAS attendees. We conclude that a BPAS/PHLS collaboration would make it feasible to monitor chlamydia prevalence in this nationwide service while offering a screening intervention that is likely to bring significant benefit to patients, their partners and, indirectly, to the wider population. More detailed examination of the potential psychosocial benefits and costs of chlamydia screening in this population, and strategies for ensuring effective partner notification and treatment, should now be explored.

\section{ACKNOWLEDGEMENTS}

We would like to thank the following: all BPAS staff who worked on the project and in particular Judith Killeby for her organising skills; staff at Liverpool and Telford PHL, particularly Rebecca Wood and Suzanne Cooke who undertook LCR processing; Fiona Warburton and Andre Charlett for the statistical analyses presented in this report; Mike Bowen, Ian Simms, and Sally Wellsteed for comments on the draft.

The study was supported by resources from BPAS, PHLS, and a grant from the Department of Health. 


\section{CONTRIBUTORS}

The project was conceived by $\mathrm{HM}$ and $\mathrm{JH}$ and arose as a progression of previous work on chlamydia testing of BPAS clients. Together with SS they were co-authors and planned the clinical and laboratory aspects of the study. IJ and CP were responsible for all planning and patient care at the BPAS. They used their management systems to select representative sites and to provide data on the timely arrival of test results. Both commented on the manuscript; KF was involved in planning for data gathering, was responsible for statistical analysis and was co-author in presenting this aspect of the paper.

Conflict of interest: None.

\section{Authors' affiliations}

H Mallinson, PHLS Laboratory, University Hospital Aintree, Lower Lane, Liverpool L9 7AL, UK

J Hopwood, Chlamydia Pilot Office, St Catherine's Hospital, Birkenhead CH42 OLQ, UK

S Skidmore, PHLS Laboratory, Princess Royal Hospital, Telford TF6 6TF, UK

K Fenton, HIV/STD Division, PHLS Communicable Disease Surveillance Centre, 61 Colindale Ave, London NW9 and Department of STDs, Royal Free and University College London Medical School, off Capper Street, London WCIE 6AU, UK

C Phillips, I Jones, British Pregnancy Advisory Service, Austy Manor, Wootton Wawen, Solihull, West Midlands B95 6BX, UK

\section{REFERENCES}

1 Simms I, Catchpole M, Brugha R, et al. Epidemiology of genital Chlamydia trachomatis in England and Wales. Genitourin Med 1997:73:122-6.

2 Department of Health. Chlamydia trachomatis: summary and conclusions of the Chief Medical Officer's Expert Advisory Group. London: DoH, 1998.

3 Moller B, Ahrons S, Laurin J, et al. Pelvic infection after elective abortion associated with Chlamydia trachomatis. Obstet Gynaecol 1982:59:210-21.

4 Skjeldestad F, Nordbo S, Hadgu A. Sentinel surveillance of Chlamydia trachomatis infection in women terminating pregnancy. Genitourin Med 1997;73:29-32.

5 Hopwood J, Mallinson H, Jones I. There is more to a test than technology-evaluation of testing for chlamydia infection in a charitable-sector termination service. Br J Family Planning 1998;23:116-19.

6 Catchpole M, Pimenta J, Rogers P, et al. Opportunistic screening for Chlamydia trachomatis: methodology and preliminary results from a UK pilot study. In: Saikku P, ed. Proceedings of the IV Meeting of the European Society for Chlamydial Research, Universitas Helsingiensis. Helsinki, Finland, August 2000:429.

7 Garland SM, Tabrizi S, Hallo J, et al. Assessment of Chlamydia trachomatis prevalence by PCR and LCR in women presenting for termination of pregnancy. Sex Transm Infect 2000;76:173-6.

8 Thompson C. Is it evidence-based practice? Prophylactic antibiotics for termination of pregnancy to minimize post-abortion pelvic infection? Int J STD AIDS 2000;11:617-8.

9 Blackwell AL, Emery SJ, Thomas PD, et al. Universal prophylaxis for Chlamydia trachomatis and anaerobic vaginosis in women attending for suction termination of pregnancy: an audit of short-term health gains. Int J STD AIDS 1999;10:508-13.

If you wish to comment on any article published in Sexually Transmitted Infections you can send an eLetter using the eletters link at the beginning of each article. Your response will be posted on Sexually Transmitted Infections online within a few days of receipt (subject to editorial screening).

\section{www.sextransinf.com}

